

\title{
Development of an electrochemical biosensor for $\Delta$ 9-tetrahydrocannabinol detection based on gold surfaces functionalized with self-assembled monolayers
}

 \\ 1 LIMA, Faculté de Médecine, Université de Monastir, Av. Avicenne Monastir - 5000 - Monastir - Tunisie. E- \\ Mails : mina.souiri@yahoo.fr (M.S.) ; ali.othmane54@gmail.com (A.O.). \\ 2 Laboratoire de Biochimie, CHU Fattouma Bourguiba, 5000-Monastir - Tunisie \\ Mails : Wahiba.douki@yahoo.fr (W.D.), saadaoui.fphm@gmail.com (M.H.S) \\ * Author to whom correspondence should be addressed; E-Mail: Amaltlili24@ gmail.com \\ Tel.: + 33758595355
}

Received: / Accepted: / Published:

\begin{abstract}
:
In this work, we have developed a new alternative for $\triangle 9$-THC detection: an electrochemical immunosensor. To the very best of our knowledge, this is the first study concerning cannabinoid detection with a biosensor. Here, gold electrodes were first functionalized with a self-assembled monolayer (SAM) of mercaptoundecanoid acid (MUDA), a long-chain carboxylic acid-terminating alkanethiol. The acid groups were then activated with the 1-ethyl-3- (3-dimethylaminopropyl) carbodiimide/N-hydroxysuccinimide (EDC/NHS) before further grafting of anti-cannabinoid monoclonal antibodies. Electrochemical results showed a wide dynamic range varying between $10^{-15}$ and $1 \mathrm{mg} / \mathrm{ml}$. The limit of detection of the designed sensor, of order of $10^{-15} \mathrm{mg} / \mathrm{ml}$, is largely inferior to that obtained with conventional techniques.
\end{abstract}

Keywords: Electrochemical immunosensor, $\Delta 9$-tetrahydrocannabinol, SAMs

\section{Introduction}

Cannabis is one of the most widely-consumed drugs in the world. According to the United Nations' Word drug report, the number of cannabis consumers worldwide was of order of 238 million in 2017 and this number is in constant increase. Certainly, cannabis can be administrated for therapeutic use, and in several medicines; in this case, the administered doses take into account both medical and psychic states of the patients. When consumed as a drug, and depending upon the dose, method of administration and prior experience, cannabis can cause an altered state of consciousness, heart rate increase, and impaired coordination / concentration. For an equivalent dose of $15 \mathrm{mg}$ of $\Delta 9$-tetrahydrocannabinol, the active ingredient of cannabis, the consumer can get attacks of anxiety, hallucinations, delusions and impression of knowing nothing ${ }^{1}$. France has recorded $7 \%$ of fatal accidents involving cannabis' user's drivers. The $\triangle 9$-THC, as an example of cannabis, can be detected with its metabolites in the blood, sweat and saliva by chromatographic methods with a limit of detection in the order of $0.4 \mathrm{ng} / \mathrm{ml}$. But, these methods are very expensive and need a high 
technicality. This study concerns the design and realization of a sensitive biosensor for quick and

\section{Results and Discussion}

\subsection{Sensor construction and electrochemical characterization}

The gold surfaces were first cleaned with a piranha solution $\left(\mathrm{H}_{2} \mathrm{SO}_{4} / \mathrm{H}_{2} \mathrm{O}_{2} ; 97 \% / 33 \%\right)$ during 7 min to obtain a clean and activated surface. The electrodes were then immersed in a MUDA solution $(20 \mathrm{mM})$ during $18 \mathrm{~h}$ at $4^{\circ} \mathrm{C}$. An acid self-assembled monolayer is thus formed through the affinity between sulfur and gold ${ }^{2}$. The acidic group were after that activated by EDC/NHS solution $(75 \mathrm{mM} / 15 \mathrm{mM})$ during $1 \mathrm{~h}$ to form a more reactive NHS-ester group ${ }^{4}$. $20 \mu \mathrm{l}$ of $1 \mathrm{mg} / \mathrm{ml}$ anti-cannabinoïde monoclonal antibody were then deposited on the functionalized surfaces. After $15 \mathrm{~h}$, the electrodes were rinsed with $\mathrm{PBS}(0.16 \mathrm{M})$ to eliminate the excess of the antibody. To block the non-reactive sites, the electrodes were incubated during $1 \mathrm{~h}$ in a BSA solution (10\%). A schematic representation of these steps is presented in Figure 1.

The different steps of surface modification, were characterized by electrochemical impedance spectrometry (in the presence of $\mathrm{Fe}(\mathrm{CN})_{6}{ }^{3-14-}(5 \mathrm{mM})$ and $\left.\mathrm{PBS}\right)$ and by angle of contact measurements.

Electrochemical results gathered in Figure 2.A show an increase in the resistance of charge transfer $\mathrm{R}_{\mathrm{tc}}$ after SAM's formation which may be attributed to the deprotonation of acidic groups, which partially block the electron transfer efficient specific detection of cannabis

towards the surface. Contact angle values gathered in Table. 1 confirm this result as the angles decreased from $72.3^{\circ}$ to $54.2^{\circ}$. In fact, after deprotonation, the surface became extremely polar which causes the spreading of the drop on the plate.

Activation with EDC/NHS leads to the decrease of the charge transfer resistance. This is probably due to the fact that after activation, the terminal carboxylic groups of MUDA were converted to neutral NHS-esters, which facilitates the charge transfer. Replacing the group NHS-ester with antibodies leads to a decrease in the $R_{t c}$ and contact angle values due to "favorable" antibodies orientation. The small increase of $R_{t c}$ after incubation in BSA may be attributed to the blocking character of this protein. The further antibodies-antigen recognition leads to $R_{t c}$ increase.

\section{2. $\triangle 9-T H C$ detection}

$\triangle 9$-THC antigen concentrations were varied from $10^{-15}$ to $1 \mathrm{mg} / \mathrm{ml}$. Figure. $2 \mathrm{~B}$ shows that an increase in $\Delta 9$-THC concentration leads to an $R_{\text {tc }}$ augmentation. This may be attributed to the steric hindrance related to the complex antibodies- antigen formation.The limit of detection of the designed sensor, of order of $10^{-15} \mathrm{mg} / \mathrm{ml}$, is largely inferior to that obtained with conventional techniques.

Table1. Contact angle measurement after each modification of the surface

\begin{tabular}{|c|c|}
\hline Surface modification & Contact angle measurements \\
\hline Naked Electrode & $72.3^{\circ}$ \\
\hline MUDA surface functionalized & $54.2^{\circ}$ \\
\hline Ab Surface modified & $12.4^{\circ}$ \\
\hline
\end{tabular}

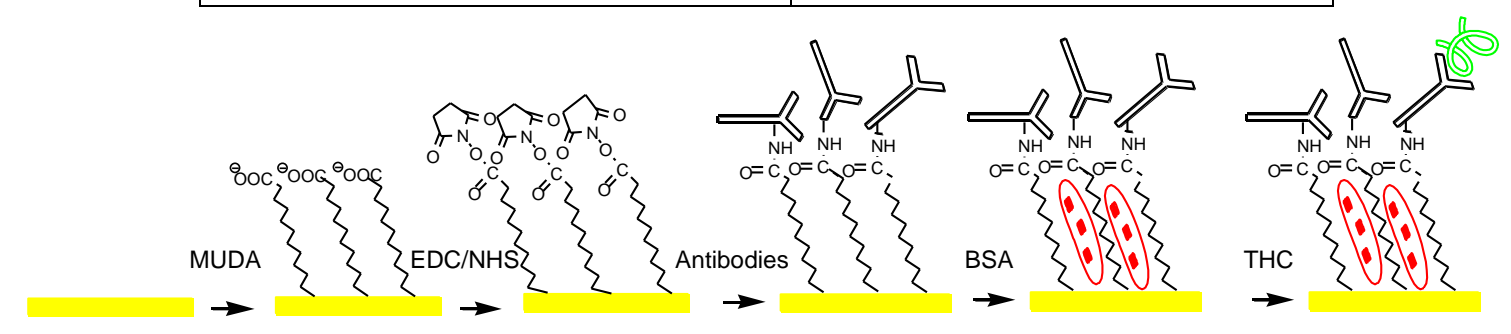

Figure1. Schematic of biosensor construction 

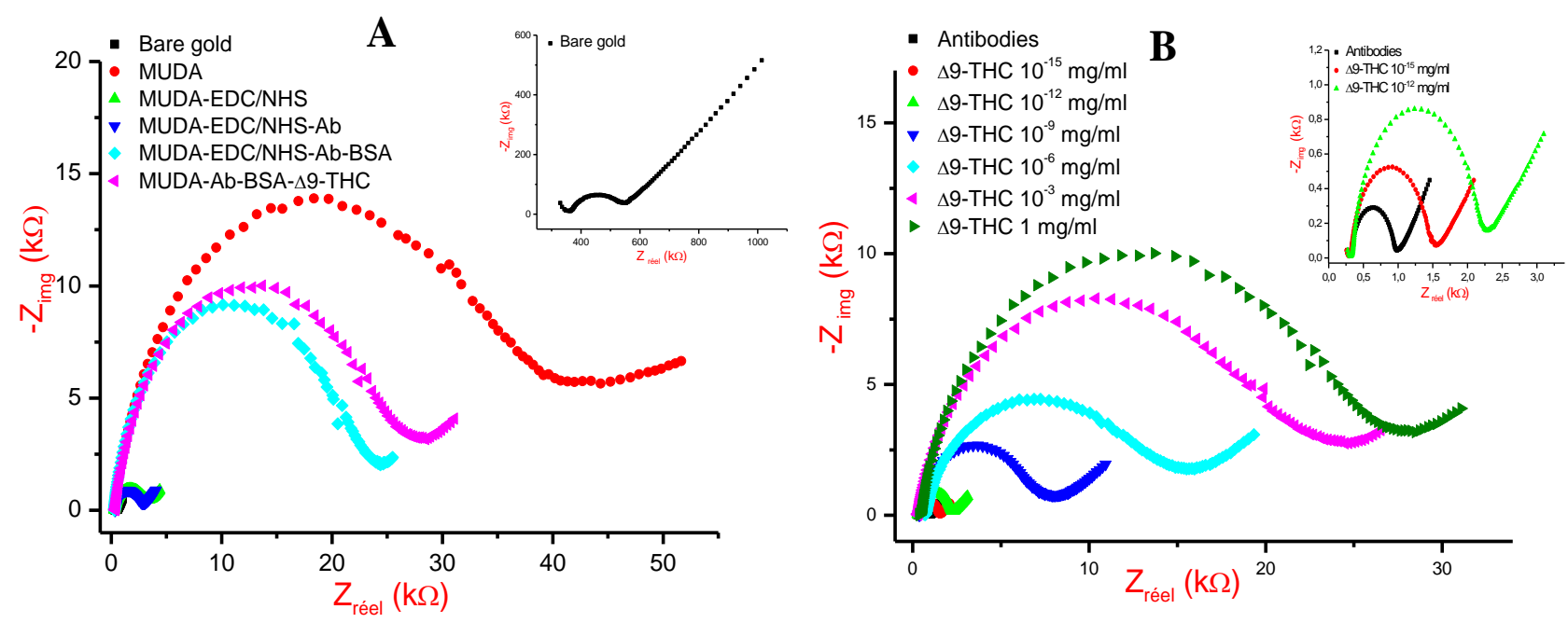

Figure2. Impedance analysisfollow up after A) the different functionalization steps of gold electrode B)

$\triangle 9$-THC detection by the designed immunosensor

\section{Materials and methods}

The $\triangle 9$-THC antigens and antibodies were purchased from. 11-Mercaptoundecanoic acid (MUDA), 1-ethyl-3-(3-dimethylaminopropyl) carbodiimide (EDC), N-Hydroxysuccimide (NHS), phosphate buffer solution (PBS) and

\section{Conclusion}

A MUDA-SAM electrochemical biosensor was designed and realized for rapid $\triangle 9$-THC detection.Electrochemical impedance spectrometry and contact angle measurements were investigated to follow up the different functionalization steps of the gold electrodes. bovine albumin serum (BSA) were purchased from sigma Aldrich. All chemical measurements were determinate with the voltalab using the calomelelectrode as reference electrode and palatine electrode as auxiliary electrode.
The designed immunosensor permits to $\operatorname{detect} \Delta 9$ THC antigen in a range of concentration varying from $10^{-15}$ to $1 \mathrm{mg} / \mathrm{ml}$. This study paves the way for the development of new analytical devices able to detect cannabis traces in few minutes and in humanbody fluids.

\section{References}

1. Institut national de la santé et de la recherche médicale (France). Cannabis - quels effets sur le comportement et la santé? (Les Editions INSERM, 2001).

2. Akram, M., Stuart, M. C. \& Wong, D. K. . Direct application strategy to immobilise a thioctic acid self-assembled monolayer on a gold electrode. Anal. Chim. Acta504, 243-251 (2004).

3. Palazon, F., Montenegro Benavides, C., Léonard, D., Souteyrand, É. \& Chevolot, Y. Carbodiimide/NHS Derivatization of $\mathrm{COOH}-$ Terminated SAMs: Activation or Byproduct Formation? Langmuir30, 4545-4550 (2014). 